Carotenoid-Based Coloration, Condition, and Immune Responsiveness in the Nestlings of a Sexually Dimorphic Bird of Prey

Author(s): Audrey Sternalski, François Mougeot, Lorenzo Pérez-Rodríguez, and Vincent

Bretagnolle

Reviewed work(s):

Source: Physiological and Biochemical Zoology, Vol. 85, No. 4 (July/August 2012), pp. 364-375

Published by: The University of Chicago Press

Stable URL: http://www.jstor.org/stable/10.1086/665981

Accessed: 18/06/2012 03:49

Your use of the JSTOR archive indicates your acceptance of the Terms \& Conditions of Use, available at http://www.jstor.org/page/info/about/policies/terms.jsp

JSTOR is a not-for-profit service that helps scholars, researchers, and students discover, use, and build upon a wide range of content in a trusted digital archive. We use information technology and tools to increase productivity and facilitate new forms of scholarship. For more information about JSTOR, please contact support@jstor.org. 


\section{Carotenoid-Based Coloration, Condition, and Immune Responsiveness in the Nestlings of a Sexually Dimorphic Bird of Prey}

\author{
Audrey Sternalski ${ }^{1,2, *}$ \\ François Mougeot ${ }^{3}$ \\ Lorenzo Pérez-Rodríguez ${ }^{4}$ \\ Vincent Bretagnolle ${ }^{2}$ \\ ${ }^{1}$ Instituto de Investigación en Recursos Cinegéticos, Consejo \\ Superior de Investigaciones Cientificas, Universidad de \\ Castilla-La Mancha, Junta de Comunidades de Castilla-La \\ Mancha, Ronda de Toledo s/n, 13005 Ciudad Real, Spain; \\ ${ }^{2}$ Centre d'Études Biologiques de Chizé, Centre National de la \\ Recherche Scientifique, 79360 Beauvoir-sur-Niort, France; \\ ${ }^{3}$ Estación Experimental de Zonas Áridas, Consejo Superior \\ de Investigaciones Cientificas, Carretera de Sacramento s/n, \\ 04120 La Cañada de San Urbano, Almería, Spain; \\ ${ }^{4}$ Departamento de Ecología Evolutiva, Museo Nacional de \\ Ciencias Naturales, Consejo Superior de Investigaciones \\ Cientificas, José Gutiérrez Abascal 2, 28006, Madrid, Spain
}

Accepted 3/27/2012; Electronically Published 6/8/2012

\begin{abstract}
In many birds, nestlings exhibit brightly colored traits that are pigmented by carotenoids. Carotenoids are diet limited and also serve important health-related physiological functions. The proximate mechanisms behind the expression of these carotenoid-pigmented traits are still poorly known, especially in nestlings with sexual size dimorphism. In these nestlings, intrabrood competition levels and growth strategies likely differ between sexes, and this may in turn influence carotenoid allocation rules. We used dietary carotenoid supplementation to test whether wild marsh harrier (Circus aeruginosus) nestlings were carotenoid limited and whether carotenoid allocation strategies varied between sexes, which differ in their size and growth strategies. When supplemented, nestlings used the supplemental carotenoids to increase their coloration independently of their sex. We showed that the condition dependence of the carotenoid level and the response to an immune challenge (phytohemagglutinin test) differed between sexes, possibly because sexual size dimorphism influences growth strategies and/ or intrabrood competition levels and access to different types
\end{abstract}

\footnotetext{
*Corresponding author; e-mail: audrey.sternalski@gmail.com.
}

Physiological and Biochemical Zoology 85(4):364-375. 2012. (C) 2012 by The University of Chicago. All rights reserved. 1522-2152/2012/8504-1142\$15.00. DOI: $10.1086 / 665981$ of food. In this species, which often feeds on mammals, a tradeoff likely exists between food quantity (energy) and quality (carotenoid content). Finally, carotenoid-based coloration expressed in marsh harrier nestlings appeared to be indicative of immune responsiveness rather than condition, therefore potentially advertising to parents nestling quality or value rather than nutritional need.

\section{Introduction}

Conspicuous carotenoid-pigmented traits are among the most used signals in social interactions (review in Espmark et al. 2000; McGraw and Hill 2006). Carotenoids are not synthesizable by vertebrates but must be ingested and are therefore often a diet-limited resource (Endler 1980; Olson and Owens 1998; Sternalski et al. 2010). In addition, carotenoids have important health-related physiological functions. They play roles in mechanisms directly or indirectly related to immune defense, and they can act as antioxidants or scavengers of free radicals produced in intense metabolic processes such as rapid growth, immune responses, and responses to stressful conditions (Møller et al. 2000; Surai 2002; Pérez-Rodríguez 2009). Under the assumption that carotenoids are a limited resource in the environment and are used in different physiological processes, possible life-history trade-offs in their allocation to competing functions have been suggested.

How carotenoid-pigmented traits evolved and are maintained as honest indicators of individual quality remains a central issue in evolutionary biology. In numerous bird species, carotenoid-pigmented traits are displayed by both adults and nestlings (e.g., Bortolotti et al. 2000; Costantini et al. 2007). However, to date, most of the attention has focused on the functions and proximate mechanisms of trait expression in adults rather than nestlings. In adult birds, carotenoid-pigmented ornaments are a common target of female choice (e.g., Hill 2002; Blount et al. 2003; Mougeot and Arroyo 2006; but see Metzger and Bairlein 2011 for alternative functions), and a trade-off has been shown to exist with allocating limited carotenoids for sexual displays versus for immune defenses (Lozano 1994; Møller et al. 2000; Faivre et al. 2003). Carotenoid-based ornaments therefore often function as reliable indicators of quality in adults (i.e., better health and/or immunocompetence; McGraw and Hill 2006; Mougeot et al. 2007, 2009; Mougeot 2008). 
Whether nestling carotenoid-based coloration honestly reveals nestling quality, as it does for adults, remains poorly known. Carotenoid-pigmented traits can inform about a nestling's condition and therefore its potential need (Loiseau et al. 2008; Dugas and McGraw 2011), or it may instead inform about a nestling' quality, such as immunocompetence (e.g., Saino et al. 2000, 2003a). Within a brood, sibling competition affects resource acquisition, including of carotenoids, and it also potentially affects resource allocation strategies. Different parental strategies of resource allocation-for example, according to sex and position in the brood hierarchy-may influence the degree of sibling competition (Mock and Parker 1997) and ultimately how nestlings use carotenoids. In species that display sexual size dimorphism, competition levels vary mainly with nestling sex, with the larger sex being a superior competitor that usually obtains more resources. Nestlings of different sexes may also require more or different specific resources according to their growth strategies (Blanco et al. 2006; Dubiec et al. 2006). As such, individual resource allocation rules for competing functions may differ between the sexes. Hence, the relative effects of carotenoid supplementation on coloration and response to immune challenge could differ between males and females. Surprisingly, despite having a relevant role in allocation strategies, the sex-specific requirements and responses of chicks to carotenoid availability, as well as potential sexual differences in carotenoid limitation levels, are still poorly known (but see Benito et al. 2011).

The marsh harrier Circus aeruginosus is a sexually dimorphic raptor species (with adult females being ca. 34\% heavier than males; Bavoux et al. 2006) that presents hatching asynchrony (i.e., ca. 2-3 d between eggs hatching), which creates a marked body-mass difference between nestlings that is related to their hatching order, with the first-hatched nestling being heavier and larger than later-hatched nestlings. In addition, the size dimorphism, although apparent at fledging, becomes detectable only from ca. $20 \mathrm{~d}$ old, when nestling females become heavier and larger than males (Bavoux et al. 2006). Female nestlings fledge at ca. 35-40 d old and male nestlings fledge at ca. 30$35 \mathrm{~d}$ old (because they are lighter). Marsh harrier nestlings display yellow carotenoid-pigmented bare parts (ceres and tarsi), similar to adults and other harrier species, as early as their first week of age (e.g., Sternalski et al. 2010, 2012b). Using dietary carotenoid supplementation, we tested whether wild nestling marsh harriers were carotenoid limited and whether they used the supplemented pigments for coloration (i.e., to increase cere and tarsi coloration), to respond to a phytohemagglutinin (PHA) challenge (Smits et al. 1999; Demas et al. 2011), or both. The PHA skin test measures an individual's proinflammatory potential (Vinkler et al. 2010) and some aspects of cellular immune responsiveness (see Martin et al. 2006; Tella et al. 2008). PHA responses are carotenoid dependent (carotenoid supplementation can enhance responsiveness; Blount et al. 2003; McGraw and Ardia 2003), so the PHA skin test is particularly useful to investigate carotenoid allocation priorities. We further tested whether carotenoid allocation priorities in nestlings differed between sexes, given size dimor- phism. We expected circulating carotenoid levels to be positively related to coloration and response to a PHA challenge, and we expected carotenoid supplementation to increase coloration and/or response to a PHA challenge (assuming a diet-carotenoid limitation). Moreover, within a brood, we expected intrabrood competition to be lower for female than for male nestlings, given their larger size. If intrabrood competition influences food acquisition and, consequently, carotenoid availability, we predicted males to be more constrained than females. We further expected different sex-specific carotenoid allocation strategies toward coloration versus a response to a PHA challenge.

\section{Methods}

\section{Carotenoid Supplementation and Field Experimental Design}

We studied a marsh harrier population located in central western France (Charente-Maritime district: $46^{\circ} 28^{\prime} \mathrm{N}, 1^{\circ} 08^{\prime} \mathrm{W}$ ). The study area (ca. $66 \mathrm{~km}^{2}$ ) is characterized by intensive farming for the cultivation of winter cereals, rape, and spring-sown crops (sunflower and corn), with pasture and other permanent or semipermanent crops grown for livestock rearing. In the study population, marsh harriers mainly feed on small mammals (in particular, common voles Microtus arvalis; $47 \%$ ) and, to a lesser extent, birds (10\%), reptiles and insects (2\%), and fish (20\%; see Sternalski et al. 2012a).

In 2006, we collected blood from three nestlings of this species to identify the circulating carotenoids, using high-performance liquid chromatography (HPLC; see Stradi et al. 1995 for a detailed description of the method). Integuments from three dead marsh harriers were obtained from recovery centers and were also analyzed to identify the carotenoids deposited there, also by using HPLC. Nestlings circulated mainly zeaxanthin and lutein (both xanthophyll pigments accounted for $83 \%$ of the total carotenoids) and, to a lesser extent, $\beta$-cryptoxanthin and $\beta$-carotene $(17 \%)$. The same carotenoids were found in similar proportions in the marsh harrier integument we sampled (ceres and tarsi).

We conducted the carotenoid supplementation experiment in June and July 2007. Harrier nests were searched for continuously during the prelaying period; we visited most of them for the first time during incubation, although in some cases they were first visited after hatching. We measured and weighed eggs to estimate hatching date (ca. \pm 2 d; see Millon et al. 2008) and to plan future nest visits to start the experiment. In our study population, the mean \pm SD hatching brood size was $4.01 \pm 1.03$ chicks per nests. During the second nest visit, a few days after hatching, chicks were weighted and measured and individually marked (first using a nontoxic marker pen code and later using metal rings) so that they could be identified throughout the experiment period. Chicks were ordered by weight, with first-hatched (older) nestlings being heavier than nestlings hatched later due to the marked hatching asynchrony displayed by the species. Nestlings' ages (and thus hatching dates) were also retrospectively estimated (ca. $\pm 2 \mathrm{~d}$ old) by using biometrics, specifically, wing and weight growth curves 
that were previously established from captive marsh harrier nestlings fed ad lib. (A. Sternalski and V. Bretagnolle, unpublished data). Carotenoid supplementation effects were tested, using a within-brood experimental design, on a total of 16 nests. Within a brood, the carotenoid treatment (control or supplemented) of the heaviest chick was randomly decided, and then treatment was sequentially assigned (alternating treatment and control) to the remaining nestlings. Thus, within each brood we had control (nonsupplemented) and treated (supplemented) nestlings, with the treatments homogenized by hatching order (see table 1 for additional information and sample size).

For supplementation, carotenoids were administered to nestlings directly in the crop, using a flexible syringe and a semiliquid form of Oro Glo, a commercially available product (Kemin, Nantes). To avoid photooxidation of pigments, the solution was kept in a refrigerated bag and in opaque containers until it was administered to the chicks. The solution contained lutein and zeaxanthin, the two main xanthophyll pigments circulated by marsh harrier nestlings, in similar proportions to those found in nature (lutein : zeaxanthin, $20: 1$; total concentration, $11 \mathrm{mg} / \mathrm{mL}$ ). In addition to these xanthophylls, the Oro Glo solution contained a very small amount of soybean oil. Therefore, a small amount of lipid (and thus energy) was also provided to supplemented nestlings, which may have facilitated carotenoid mobilization and transport for supplemented chicks (lipids can facilitate carotenoid transport; Stevens 1996). We did not provide soybean oil only to control nestlings (as was done in several previous studies: Alonso-Alvarez et al. 2004; Blount and Matheson 2006; Ewen et al. 2008), in order to avoid its possible effects on carotenoid acquisition and mobilization in control chicks and also because the amount of soybean oil included was considered to be very small compared with the daily food intake of all nestlings (which are regularly fed every 1-2 h with energy-rich natural prey, such as voles, reptiles, and birds).

Our initial aim was to provide supplementation to nestlings three times between $10 \mathrm{~d}$ of age and fledging (chicks less than $10 \mathrm{~d}$ old were considered too young and too small to receive treatment), every $5 \mathrm{~d}$, with nestlings receiving increasing amounts of carotenoids of 22, 44, and $66 \mathrm{mg}$ on first, second, and third visits (when chicks were ca. 15, 20, and $25 \mathrm{~d}$ old), respectively. Little is known about the daily dose of carotenoids consumed by harrier nestlings in nature. We selected our doses on the basis of previous studies conducted on kestrel nestlings (see Casagrande et al. 2007) and nestlings of the closely related Montagu's harrier (see Sternalski et al. 2010). We adjusted doses used in these studies to the body masses of marsh harrier nestlings. Thus, because carotenoid doses were provided according to nestling body size (nestling age being estimated a posteriori), some nestlings may have received the same dose on two separate occasions instead of three increasing doses of 22, 44, and 66 mg. In addition, because the number of supplementations administered depended on (1) nestling age at the first supplementation and (2) fieldwork constraints (we tried to minimize the number of nests visits and disturbances to nests), some nestlings received only two carotenoid supplementations instead of three (e.g., they already fledged or were not found during the last nest visit). Therefore, the two carotenoid-supplemented groups differed in age (i.e., nestlings that received three supplementations were on average younger that those that received two) and total number of supplementations received. Within the treated groups, eight nestlings received two supplementations (total dose of $66 \pm 33 \mathrm{mg}$ of carotenoids at $14 \pm 3$ and $22 \pm 3 \mathrm{~d}$ of age, corresponding to 0.14 and 0.10 $\mathrm{mg} / \mathrm{g}$ for 22-d-old male and female nestlings, respectively) and 11 nestlings received three supplementations (total dose of $154 \pm 55 \mathrm{mg}$ of carotenoids at $11 \pm 3,18 \pm 3$, and $25 \pm 3 \mathrm{~d}$ of age, corresponding to 0.30 and $0.22 \mathrm{mg} / \mathrm{g}$ for 25 -d-old male and female nestlings, respectively). We thus considered two carotenoid supplementation levels (hereafter referred as to 2SUP and 3SUP treatments) a posteriori according to the number of supplementations and the doses that nestlings received.

On the first visit (before treatment), we took a blood sample and measured (1) body mass (using a Pesola scale; to the nearest $1 \mathrm{~g}$ ), (2) wing length (using a ruler; to the nearest $1 \mathrm{~mm}$ ), (3) tarsus length (using a caliper; to the nearest $0.1 \mathrm{~mm}$ ), and (4) coloration of bare parts (cere and tarsi; using a colorimetric chart; see below). When chicks were close to fledging (i.e., on last nest visit; nestling age, $30 \pm 2 \mathrm{~d}$ ), we collected another blood sample and again measured body mass, wing length,

Table 1: Sample sizes and breeding parameters according to the experimental treatment (no carotenoid supplementations vs. carotenoid supplementations)

\begin{tabular}{lcc}
\hline & \multicolumn{2}{c}{ Experimental treatment } \\
\cline { 2 - 3 } Parameters & $\begin{array}{c}\text { No carotenoid } \\
\text { supplementations }\end{array}$ & Carotenoid supplementations \\
\hline No. nestlings & $28(11$ males vs. 17 females $)$ & 24 (13 males vs. 11 females $)$ \\
Brood size & $4.11 \pm 1.07$ & $4.04 \pm 1.00$ \\
Age before treatment & $13.7 \pm 4.6$ & $13.2 \pm 3.7$ \\
Age after treatment & $29.9 \pm 3.1$ & $30.3 \pm 2.7$ \\
Sex ratio & .39 & .54 \\
\hline
\end{tabular}

Note. Parameters are number of nestlings per experimental treatment (and sex), average brood size per treatment, average nestling age before and after experimental treatment, and average sex ratio per treatment. Data are presented as means $\pm \mathrm{SD}$. A total of 16 nests were used for the experiments. 
tarsus length, and bare-parts coloration. At that time we also measured response to a PHA challenge (see below). Blood was taken from the brachial vein with a heparinized capillary, kept refrigerated $\left(0^{\circ}-5^{\circ} \mathrm{C}\right)$, and centrifuged at $10,000 \mathrm{rpm}$ within 4 $\mathrm{h}$ of collection. Plasma samples were stored at $-20^{\circ} \mathrm{C}$ until quickly analyzed. Pellets were used for molecular sexing of nestlings, following Fridolfsson and Ellegren (1999), using polymerase chain reaction amplification and agarose electrophoresis and the primer sequence of an intron fragment that is clearly different in size between male and female nestlings.

\section{Color Measurements}

Cere and tarsi colorations were measured by direct comparison with a yellow-orange colorimetric chart (Yolk Color Fan, Roche, Neuilly-sur-Seine, France), a method previously used and validated for the Montagu's harrier Circus pygargus (see Sternalski et al. 2010). Cere and tarsi color scores were summed to obtain a nestling coloration score (ranging from 0 , i.e., very pale yellow, to 10, i.e., intense yellow) indicative of overall carotenoidbased coloration (see Sternalski et al. 2010).

\section{Carotenoid Assays}

We followed a general procedure used previously with harriers (Sternalski et al. 2010, 2012b) as well as with other species (e.g., Mougeot et al. 2010). Briefly, carotenoid concentration in plasma was determined, using a spectrophotometer. Plasma samples were diluted in acetone ( $1: 6$ dilution), and the mixture was vortexed and centrifuged at $10,000 \mathrm{~g}$ for $5 \mathrm{~min}$ to precipitate the flocculent proteins. The optical density of the supernatant was examined at $450 \mathrm{~nm}$, using microtiter plates and a Biotek Powerwave XS2 spectrophotometer (Winooski, VT). Plasma carotenoid concentrations $(\mu \mathrm{g} / \mathrm{mL})$ were calculated, using a lutein standard curve (Extrasynthese, ref. $0306 \mathrm{~S}$ ). Intraand interplate repeatabilities were estimated from a random subset of samples measured twice (intraplate: $F_{14,15}=26.3$, $P<0.001, r=0.92$; interplate: $F_{54,55}=10.3, P<0.001, r=$ $0.83)$.

\section{Response to the PHA Challenge}

We used the PHA skin test to measure some aspects of nestling cellular immunity and proinflammatory potential (Martin et al. 2006; Tella et al. 2008; Vinkler et al. 2010). This test consists of an intradermal injection of PHA, which produces a prominent perivascular accumulation of T-lymphocytes followed by macrophage infiltration (Goto et al. 1978). It produces a small but measurable swelling, the magnitude of which indicates aspects of an individual's ability to mount a cell-mediated immune response. Each nestling was injected at a marked site on the wing web with $0.5 \pm 0.1 \mathrm{mg}$ of PHA (L8754, Sigma) suspended in $0.1 \mathrm{~mL}$ of phosphate buffer solution (PBS). Because we worked with a wild, ground-nesting species, we chose to use the simplified version of the PHA skin test (only one injection with PBS and PHA) that was developed and validated by Smits et al. (1999). This allowed us to decrease handling time and reduce nestling stress and predation risk. We measured web thickness to the nearest $0.01 \mathrm{~mm}$ at the injection site, using a pressure-sensitive dial thickness gauge (Teclock SI-112). Web thickness was measured three times before injection and $24 \mathrm{~h}$ after injection, when it was likely that the first phase of the response was mainly underway and infiltration by $\mathrm{T}$-cells was likely to be a relatively minor but increasing component of the response (Martin et al. 2006; Tella et al. 2008; Demas et al. 2011). Initial $\left(r=0.86, F_{39}=18.75, P<0.001, n=120\right)$ and final $\left(r=0.95, F_{39}=54.44, P<0.001\right)$ measurements of wingweb thickness were repeatable. We calculated response to the PHA challenge as the change after $24 \mathrm{~h}$ in average thickness (in $\mathrm{mm}$ ) at the injection site (Smits et al. 1999).

\section{Statistical Analyses}

All statistical analyses were performed using SAS 9.1 (SAS 2001). We used a general linear mixed-models procedure (MIXED; SAS) to test the effect of nestlings sex and treatment on coloration, circulating carotenoids, response to a PHA challenge, and condition index. The carotenoid treatments were broken down into two variables. The first (TREAT) contrasted the control (nonsupplemented: 0SUP) and supplemented (2SUP, 3SUP) nestlings, while the second (NSUP) contrasted carotenoid supplementation levels (2SUP vs. 3SUP). This was done by coding TREAT as $-2,1$, and 1 for control (OSUP), 2SUP, and 3SUP nestlings, respectively, and NSUP as $0,-1$, and 1 for 0SUP, 2SUP, and 3SUP nestlings, respectively. By coding variables in this manner (where the sum of each is equal to 0 ), both variables (although correlated) can be simultaneously entered in the same model, allowing us to contrast the carotenoid treatment and dose effects (see Mougeot et al. 2003 for a similar analysis). A nestling's condition index was calculated as the residuals of a generalized linear model (GLM; SAS 2001) of log-transformed body mass on age and age squared (age ${ }^{2}$; quadratic relationship with age; age: $F_{1,51}=$ 717.98, $P<0.001$; age $\left.^{2}: F_{1,51}=49.98, P<0.001\right)$ and log-transformed wing and tarsus lengths (as indexes of nestling size; GLM of log-transformed wing length: $F_{1,51}=20.99, P<0.001$; of log-transformed tarsus length: $\left.F_{1,51}=36.68, P<0.001\right)$. When we tested for treatment effects, initial models included sex, TREAT, NSUP, and all of the interactions between these explanatory variables. When we investigated the relationships between the variables, we used similar models and tested whether these relationships differed between treatment groups (i.e., TREAT, supplemented vs. nonsupplemented) according to the total number of supplementations received (i.e., NSUP) or between male and female nestlings. In addition, all models included the variable age as a fixed effect and the variable "nest" (encoding nestlings from the same brood) as a random effect. Nonsignificant $(P>0.05)$ terms were removed from the models, starting with interactions, following a backward stepwise procedure, until only the significant explanatory variables or interactions remained. The Satterthwaite correction was used to approximate the degrees of freedom. When a significant in- 
teraction between fixed factors was found, the statistical significance of each factor at different levels were computed, using the LSMEANS statement (SAS 2001). All tests were two tailed.

\section{Results}

Sex-Specific Carotenoid Allocation on Coloration, Carotenoid Level, and Condition Index

We first assessed the effects of carotenoid treatment and number of supplementations received on coloration score, carotenoid level, and condition index separately, and then we tested whether these treatment effects differed between the sexes. Before the treatments started (nestlings aged $13 \pm 4 \mathrm{~d}$ ), neither coloration score nor circulating carotenoid levels differed between male and female nestlings (see table 2), but females were in relatively better condition (relatively heavier for a similar size) than males (least square means $[\mathrm{LSM}] \pm \mathrm{SE}$ of $0.04 \pm$ 0.02 and $-0.06 \pm 0.02$, respectively; table 2 ). There were no significant differences observed between experimental groups for carotenoid levels (i.e., both for TREAT and NSUP variables) and condition index (see table 2), but a marginal one was found for coloration score (LSM $0.52 \pm 0.27$ and $1.23 \pm 0.30$ for control and supplemented nestlings, respectively; table 2). Overall, we had no evidence that the brood sex ratio differed between treatment groups (see table $1 ; \chi_{1}^{2}=1.15, P=0.28, n=52$ ).

After treatment, when nestlings were close to fledging (aged $30 \pm 3 \mathrm{~d}$ ), variation in coloration score was explained by sex and carotenoid treatment and marginally by number of supplementations received but not by any interaction between these factors (see table 2). Coloration scores were greater overall in male than in female nestlings (fig. 1a) and greater in supplemented than in nonsupplemented nestlings (fig. 1a), and they tended to be greater in nestlings that received three rather than two supplementations (LSM $7.70 \pm 0.52$ and $6.21 \pm$
0.56 for 3NSUP and 2NSUP groups, respectively). Circulating carotenoid levels were explained by sex and carotenoid treatment but not by number of supplementations received or any interaction between these factors (see table 2). Male nestlings circulated more carotenoids than female nestlings (fig. $1 \mathrm{~b}$ ), and carotenoid levels were higher in supplemented than in nonsupplemented nestlings (fig. 1b). Variation in condition index (body mass corrected for age and size) was explained by sex but not by carotenoid treatment, number of supplementations received, or any interaction between these factors (see table 2). Female nestlings were in relatively better condition than male nestlings (fig. 1c).

\section{Relationships between Coloration, Carotenoid Level, Condition Index, and PHA Challenge Response}

We further investigated associations between study variables and whether these relationships were influenced by carotenoid treatment, number of supplementations received, or nestling sex. Integumentary (i.e., cere and tarsi) carotenoid-based coloration is expected to increase with circulating carotenoid levels, and because carotenoids must be acquired via diet, a wellfed nestling is expected to be both less carotenoid limited and in better condition. We thus investigated the relationship between circulating carotenoid levels and nestling coloration and condition index in the same model. The relationship between carotenoid levels and nestling coloration score marginally depended on carotenoid treatment (MIXED model, with nest as a random effect; coloration: $F_{1,12}=0.69, P=0.422$; slope \pm SE, $0.68 \pm 0.61$; coloration $\times$ TREAT interaction: $F_{1,18}=$ $4.13, P=0.058$; see fig. $2 A$ ) but was not affected by the number of supplementations received (coloration $\times$ NSUP interaction: $F_{1,10}=0.41, P=0.537$ ) or sex (coloration $\times$ sex interaction: $\left.F_{1,9}=0.03, \quad P=0.875\right)$. Carotenoid levels and coloration

Table 2: Effects of carotenoid treatment and nestling sex on carotenoid-based coloration, circulating carotenoid level $(\mu \mathrm{g} / \mathrm{mL})$, and condition index before and after carotenoid treatment

\begin{tabular}{|c|c|c|c|c|c|c|c|c|c|}
\hline \multirow[b]{2}{*}{ Source of variation } & \multicolumn{3}{|c|}{ Coloration score } & \multicolumn{3}{|c|}{ Carotenoid level } & \multicolumn{3}{|c|}{ Condition index } \\
\hline & df & $F$ & $P$ & df & $F$ & $P$ & df & $F$ & $P$ \\
\hline \multicolumn{10}{|l|}{ Before treatment: } \\
\hline Age & 1,23 & 2.25 & .147 & 1,19 & 2.51 & .130 & 1,22 & .00 & .981 \\
\hline Sex & 1,23 & .15 & .701 & 1,19 & 2.39 & .139 & 1,22 & 14.84 & $<.001$ \\
\hline TREAT & 1,23 & 4.13 & .054 & 1,19 & .03 & .865 & 1,22 & .10 & .750 \\
\hline NSUP & 1,23 & .71 & .407 & 1,19 & .13 & .724 & 1,22 & .0 & .970 \\
\hline \multicolumn{10}{|l|}{ After treatment: } \\
\hline Age & 1,20 & 4.19 & .054 & 1,18 & .11 & .749 & 1,20 & 1.62 & .217 \\
\hline Sex & 1,20 & 9.41 & .006 & 1,20 & 6.11 & .023 & 1,21 & 13.7 & .001 \\
\hline TREAT & 1,20 & 58.14 & $<.001$ & 1,20 & 13.99 & .001 & 1,19 & .02 & .877 \\
\hline NSUP & 1,19 & 3.86 & .064 & 1,17 & .06 & .815 & 1,17 & .38 & .547 \\
\hline
\end{tabular}

Note. Because there were initial differences in coloration score between control and supplemented nestlings, we controlled initial coloration score in our analysis of color variation after treatment by including initial color score (mixed model with nest as a random effect; $F_{1,20}=9.35, P=0.006$ ) as a covariate in the model. Significant effects $(P<0.05)$ are highlighted in bold. TREAT, model contrasting control and supplemented nestlings; NSUP, model contrasting carotenoid supplementation levels. 


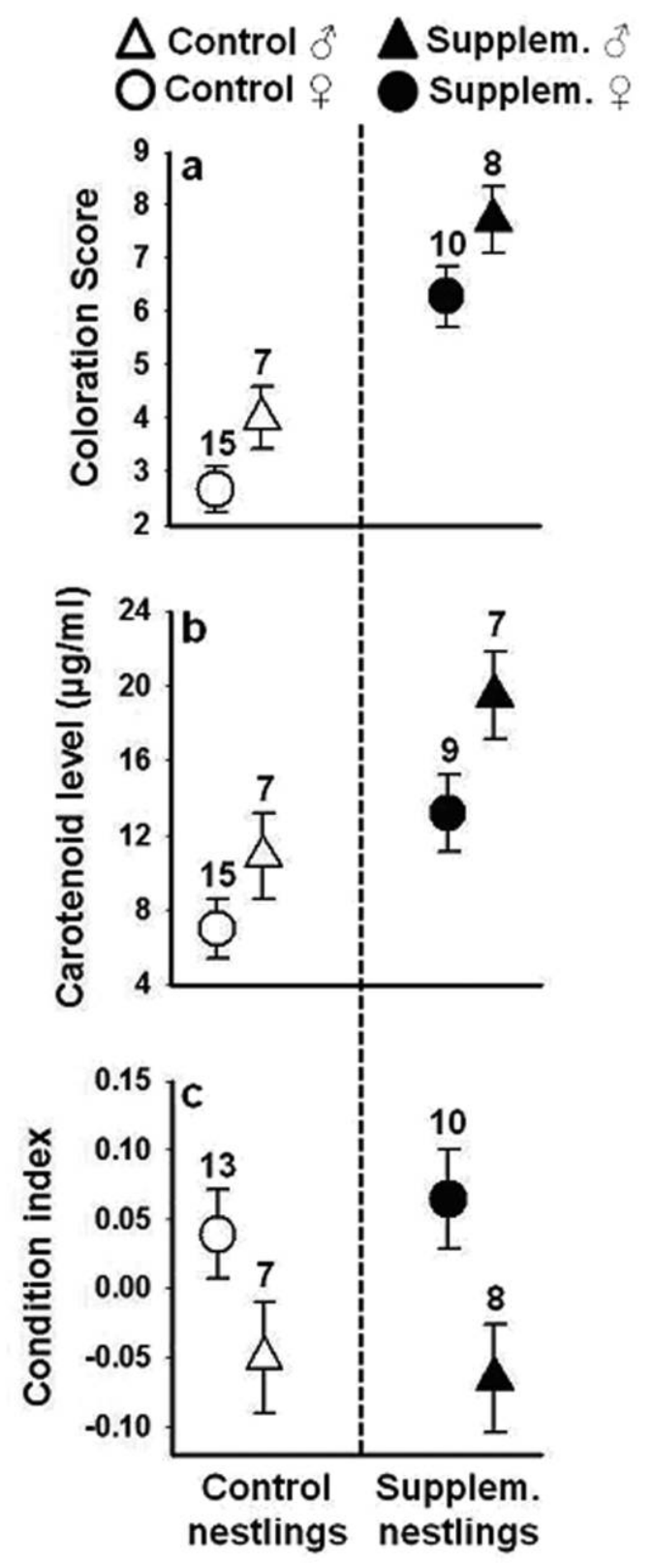

Figure 1. Means $\pm \mathrm{SE}$ (symbols + error bars) variations in $(a)$ carotenoid-based coloration score (see "Methods"), (b) circulating carotenoid level, and $(c)$ condition index according to carotenoid treatment (unfilled symbols, control nestlings; filled symbols, supplemented nestlings) and nestling sex (circles, females; triangles, males). Sample size above bars refers to the number of nestlings, with a total of 16 experimental nests. The condition index was calculated as body mass corrected for nestling age and size (see "Methods").

tended to be positively correlated in control nestlings $\left(F_{1,5}=\right.$ 3.43, $P=0.123$; slope $\pm \mathrm{SE}, 0.88 \pm 0.47)$ but negatively correlated in supplemented nestlings $\left(F_{1,4}=0.39, P=0.564\right.$; slope $\pm \mathrm{SE},-0.86 \pm 1.38)$. The marginal coloration $\times$ TREAT interaction therefore reflected opposite tendencies in control and supplemented nestlings in terms of the relationship be- tween carotenoid levels and coloration. We also found that carotenoid levels overall correlated negatively with condition index $\left(F_{1,12}=6.60, P=0.025\right.$; slope $\left.\pm \mathrm{SE},-47.50 \pm 11.44\right)$. Moreover, this relationship depended on nestling sex (condition $\times$ sex interaction: $F_{1,12}=13.51, P=0.003$; fig. $2 B$ ) and number of supplementations received (condition $\times$ NSUP interaction: $F_{1,12}=7.05, P=0.021$; fig. $2 B$ ). In males, carotenoid level negatively correlated with condition index $\left(F_{1,3}=11.98\right.$, $P=0.041$; slope $\pm \mathrm{SE},-58.48 \pm 16.90$; fig. $2 B)$, whereas in females no relationship was found $\left(F_{1,6}=2.28, P=0.182\right.$; slope \pm SE, $15.94 \pm 10.56$; fig. $2 B$ ). In addition, carotenoid levels tended to decrease with condition index in control nestlings $\left(F_{1,3}=7.38, P=0.073\right.$; slope $\left.\pm \mathrm{SE},-17.24 \pm 6.35\right)$ and in supplemented nestlings that received two supplementations (slope \pm SE, $-59.52 \pm 25.19$ ) but not in supplemented nestlings that received three supplementations (slope $\pm \mathrm{SE}$, $6.70 \pm 22.99)$.

Nestling carotenoid-pigmented traits might reveal information about nestling condition (if so, a positive relationship between theses two variables is expected) or about nestling immunocompetence (positive relationship between coloration and response to the PHA challenge). In marsh harrier nestlings, condition index was not significantly related to coloration score (MIXED model with nest as a random effect; $F_{1,21}=0.04$, $P=0.842$; slope $\pm \mathrm{SE},-0.01 \pm 0.01$ ), irrespective of carotenoid treatment (coloration $\times$ TREAT interaction: $F_{1,17}=$ $0.35, P=0.563$ ), number of supplementations received (coloration $\times$ NSUP interaction: $\left.F_{1,15}=0.08, P=0.779\right)$, or nestling sex (coloration $\times$ sex interaction: $F_{1,19}=0.93, P=$ 0.346). However, coloration score and response to the PHA challenge were positively correlated $\left(F_{1,23}=4.77, P=0.039\right)$, with the relationship being similar for both treatment groups (coloration $\times$ TREAT interaction: $F_{1,17}=0.24, P=0.634$ ) irrespective of the number of supplementations received (coloration $\times$ NSUP interaction: $F_{1,20}=0.44, P=0.513$ ) or nestling sex (coloration $\times$ sex interaction: $F_{1,19}=0.40, P=$ $0.534)$. Thus, more colored nestlings were not in better condition but mounted a greater response to PHA (slope \pm SE, $8.34 \pm 3.82)$.

Finally, we investigated whether response to the PHA challenge differed nestling sex, carotenoid-treatment group, and number of supplementations received and whether the response was related more to carotenoid level or condition index, given the unexpected (negative) relationship between both and the differences between the sexes. Overall, variation in response to the PHA challenge was explained by the condition index (MIXED model with nest as a random effect; $F_{1,14}=5.97$, $P=0.028)$ and marginally explained by carotenoid treatment $\left(F_{1,14}=4.02, P=0.065\right)$ but was not explained by nestling sex $\left(F_{1,14}=1.94, P=0.185\right)$, carotenoid levels $\left(F_{1,14}=0.60, P=\right.$ $0.453)$, or number of supplementations received $\left(F_{1,13}=1.03\right.$, $P=0.328)$. Response to the PHA challenge decreased with condition index (slope \pm SE, $-510.75 \pm 177.22$ ) and tended to be greater in supplemented than control nestlings (LSM \pm $\mathrm{SE}, 2.18 \pm 0.17$ and $1.68 \pm 0.19 \mathrm{~mm}$, respectively). In addition, the relationship between response to PHA and condition index 

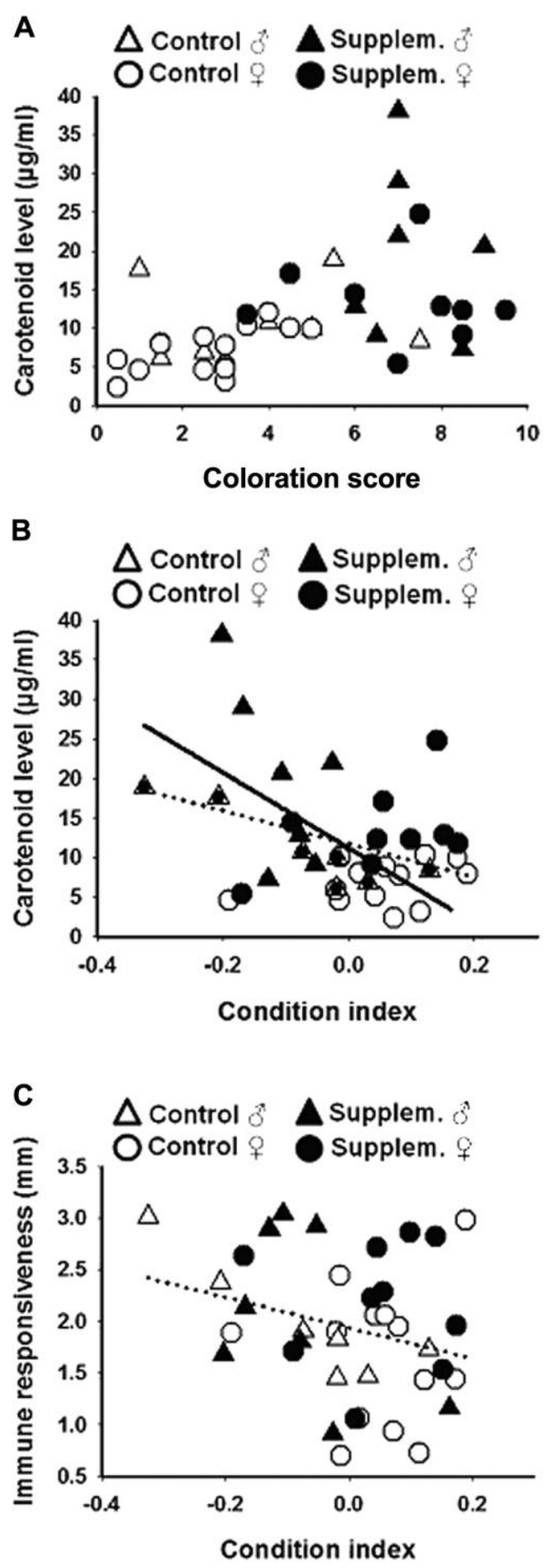

Figure 2. Relationships between circulating carotenoid levels and carotenoid-based coloration (i.e., coloration score; $A$ ) and between condition index and $(B)$ circulating carotenoid level and $(C)$ response to a phytohemagglutinin (PHA) challenge (wing-web swelling response to PHA) according to carotenoid treatment (open symbols, control nestlings; filled symbols, supplemented nestlings) and nestling sex (circles, females; triangles, males). Condition index was calculated as body mass corrected for nestling age and size (see "Methods"). Dotted lines represent fitted linear regressions for all nestlings, and solid lines (in $B$ and $C$ ) represent fitted linear regressions for male nestlings. significantly differed between the sexes (condition $\times$ sex interaction: $F_{1,14}=4.58, P=0.050$; fig. $2 C$ ), but the relationship between response to PHA and carotenoid level only slightly tended to differ between sexes (carotenoid level $\times$ sex interaction: $F_{1,14}=3.60, P=0.078$ ). In male nestlings, response to PHA tended to decrease with increasing condition index $\left(F_{1,3}=7.52, P=0.071\right.$; slope $\pm \mathrm{SE},-3.27 \pm 1.19$; fig. $\left.2 C\right)$, whereas in female nestlings, response to PHA was not related to condition index $\left(F_{1,7}=0.01, P=0.934\right.$; slope $\pm \mathrm{SE}$, $12.77 \pm 149.58$; fig. $2 C)$.

\section{Discussion \\ Effect of Carotenoid Treatment on Coloration, Carotenoid Level, Condition Index, and PHA Challenge Response}

Dietary carotenoid supplementation increased overall carotenoid availability, as shown by the higher coloration and levels of circulating carotenoids in supplemented nestlings before fledging as compared with controls. These experimental results indicate that the yellow carotenoid-based coloration and circulating carotenoid levels of wild marsh harrier nestlings do not reach their physiological thresholds (i.e., upper limits) when they are fed by parents in nature. This is consistent with the carotenoid-limitation hypothesis (Endler 1980; Hill 1992) and with previous studies conducted on other raptor species (e.g., common kestrel Falco tinnunculus: Casagrande et al. 2007; Montagu's harrier: Sternalski et al. 2010, 2012b). We also found a slight dose-dependent effect of carotenoid supplementation on nestling coloration (but not on circulating carotenoid level). Coloration tended to increase more in nestlings that received three supplementations than in those that received only two supplementations. The absence of a dose-dependent effect on circulated levels of carotenoids may be because the ingested carotenoids were rapidly diverted to body stores (including pigmented integuments) instead of being maintained in the bloodstream.

Despite increasing carotenoid availability, the carotenoid treatment did not affect nestling condition index and only marginally increased response to a PHA challenge, even at the higher supplementation doses. A lack of carotenoid supplementation effect on nestling condition index has also been shown in nestlings of other raptor species (e.g., Casagrande et al. 2007; Costantini et al. 2007; Sternalski et al. 2010). In addition, this result indicated that the carotenoid doses provided to nestlings during the experiment were not detrimental (see Olson and Owens 1998), at least with regard to body condition. In adult birds there is extensive evidence that carotenoid supplementation enhances cellular immune responsiveness (e.g., Blount el al. 2003; McGraw and Ardia 2003; Aguilera and Amat 2007; McGraw and Ardia 2007). In nestlings, however, an immunostimulant effect of carotenoids is more controversial, with some studies supporting it (Fenoglio et al. 2002; Saino et al. 2003b; Cucco et al. 2006a, 2006b) and others finding no effect (Navara and Hill 2003; Biard et al. 2006; Saino et al. 2008; Benito et al. 2011). This might be because response to a PHA challenge is not prioritized when available carotenoids increase 
or because developing chicks may not always prioritize responsiveness to PHA over other carotenoid-demanding functions. Alternatively, an effect on response to a PHA challenge might depend on the type of carotenoid supplemented, with xanthophylls having lower immunoenhancing properties than other carotenoids such as $\beta$-carotene (Bendich 1991; Fitze et al. 2007; see also Navara and Hill 2003; Cucco et al. 2006b). However, the above-mentioned studies with support for the immunostimulant effect of carotenoids in chicks (e.g., Fenoglio et al. 2002; Saino et al. 2003 b) used supplementation with xanthophylls (mostly lutein and zeaxanthin), and not $\beta$-carotene. Xanthophylls are by far the most abundant carotenoids in the plasma and body tissues of most bird species, whereas $\beta$-carotene is relatively abundant in only some species, whose diets are particularly rich in this carotenoid type (McGraw and Hill 2006). Even so, these carotenoids could be metabolized in vivo to procure the necessary resources for boosting cellular immune responsiveness (Blas et al. 2006; McGraw and Ardia 2007; Pérez-Rodríguez et al. 2008). Here we did not find a marked immunostimulant effect of carotenoid supplementation but only a weak, marginally significant one. Therefore, our results suggest that the carotenoid allocation priority is more likely toward coloration rather than responsiveness to a PHA challenge, although further work is needed to confirm this. In addition, we must remain cautious when interpreting our results, because our sample size was very small, which might have prevented us from finding significance in several statistical tests, such as this one.

\section{Sex-Specific Condition Dependence of Coloration and Carotenoid Level and Consequences to PHA Challenge Response}

Carotenoid-based coloration is expected to increase with circulating carotenoid levels, and both coloration and carotenoid levels are generally expected to be condition dependent (McGraw and Hill 2006). In this study, we found the expected positive relationship between coloration and carotenoid levels, although the relationship tended to depend on carotenoid treatment and was apparent only in control and not in supplemented nestlings. Many studies have reported such positive associations between these two parameters in adults (e.g., Hill et al. 1994; McGraw et al. 2003; Martínez-Padilla et al. 2007; Pérez-Rodríguez 2008) as well as nestlings (Tschirren et al. 2003; Thorogood et al. 2008; Casagrande et al. 2009; Sternalski et al. 2010), and our findings are thus in accordance with these previous studies. However, we did not find that coloration was condition dependent. Unexpectedly, we found an overall negative relationship between carotenoid level and condition index. This contrasts with the findings of other studies involving nestling birds (e.g., Tschirren et al. 2003; Biard et al. 2006). Most of these other studies were conducted on passerine birds that feed their nestlings a relatively carotenoid-rich diet. In these species, a positive association between overall food intake and carotenoid intake exists, hence leading to a positive condition dependence of carotenoid levels. The marsh harrier is a gen- eralist raptor (Clark 1995), but in intensified agricultural areas such as in our study site it mainly feeds on small mammals and less so on birds or insects (Sternalski et al. 2012a). Small mammals are rich in energy but poor in carotenoids, unlike other prey such as birds, reptiles, or insects (Goodwin 1984; see also Casagrande et al. 2006 for data on carotenoid content in another vole species, Microtus savii). In such situations where diet composition is diverse, there might be a reversed association between the energetic and carotenoid contents of food items that might result in a negative relationship between carotenoid level and condition index.

The negative relationship between carotenoid level and condition index was apparent only in males and in nestlings that received zero (i.e., control) or two supplementations, but it was nonexistent in females and nestlings that received three supplementations. We speculate that this might be the result of different levels of carotenoid availability caused by either different doses of provided carotenoid supplementation or different levels of intrabrood competition depending on nestling sex. Indeed, when carotenoids were highly available through the highest dose of carotenoids (i.e., 3NSUP), the negative relationship between carotenoid level and condition disappeared, reinforcing the idea that this relationship occurs via a negative association between energy and carotenoid content in prey. In addition, in species with a marked sexual size dimorphism in nestlings, the larger sex is a superior competitor that controls food access and obtains more resources: the amount of food lighter nestlings consume is the amount that larger nestlings are willing to concede, and eventually, lighter nestlings simply take whatever is left by others (Mock and Parker 1997). In marsh harriers, females are larger and heavier than males and could therefore control, to some extent, food distribution among nestlings. Females thus have access to a more varied diet, and it is possible that they preferentially consumed large prey (mammals), which are energy rich but carotenoid poor, and left the smaller prey, such as birds or insects, which are not as energy rich but are carotenoid rich, to males. This could also explain why females were in overall better condition but circulated fewer carotenoids and were less colored than males, and why males with fewer circulating carotenoids were also in better condition. An alternative explanation might be due to differences in growth strategies between male and female nestlings of this sexually dimorphic species. For instance, if females (the larger sex) invest more in growth, they may have to use more carotenoids for physiological activities related to combating oxidative stress generated by processes associated with rapid growth, such as cell proliferation (Møller et al. 2000; Surai 2002; Pérez-Rodríguez 2009). This would also explain why females express paler coloration and circulate fewer carotenoids than males and why those males with greater mass gain (greater condition index) have fewer carotenoids.

Many studies have demonstrated that response to a PHA challenge depended on circulating carotenoid levels (Blount et al. 2003; McGraw and Ardia 2003; Pérez-Rodríguez et al. 2008; Mougeot et al. 2009) and/or condition index (Saino et al. 1997; Alonso-Alvarez and Tella 2001). However, the negative rela- 
tionship between carotenoid level and condition index observed in marsh harrier nestlings may have important implications for nestling immune status. For instance, in species that preferentially feed their nestlings with carotenoid-poor food, nestlings in relatively good condition could be less responsive to a PHA challenge (because of a lower carotenoid availability in this food type), as found in our study. Alternatively, a possible trade-off between growth and immunity may exist in developing nestlings (e.g., Soler et al. 2003; Brommer et al. 2004). This could also explain some of the unexpected results, specifically, for species with sexual size dimorphism at the nestling stage, such as the marsh harrier. It is possible that females (the larger sex) invest more carotenoids in growth to the detriment of investment in immune responsiveness. This could explain the overall negative relationship between condition index and response to the PHA challenge and why male nestlings in better condition tended to mount a lower response to the PHA challenge than those in relatively poorer condition, whereas in females this relationship was nonexistent. The growth strategy of males could allow them to invest relatively more carotenoids in immune responsiveness, and within males, those investing more in growth would experience lower immune response. This suggests that under certain circumstances, the response to a PHA challenge covaries more closely with carotenoid levels than with condition index, such as in our particular system. We therefore suggest that the relationship between carotenoid levels, condition index, and response to the PHA challenge might depend on both the food restriction levels imposed by intrabrood competition and the different growth strategies of male and female nestlings.

This raises an interesting evolutionary question of how carotenoid-based traits expressed in marsh harrier nestlings evolved through signaling theory if they are not reliable condition indicators. We propose that such traits might have evolved because despite the unexpected negative relationship between circulating carotenoid levels and body condition, carotenoid-based traits are indicative of the immune status of nestlings (at least with regard to a PHA challenge), with more colored nestlings having greater immune responsiveness. Our results thus open up interesting perspectives in order to better evaluate how the relationship between carotenoid level and condition changes depending on pigment availability in th $\rightarrow$ food consumed, and growth strategies and how they influence the immune status of nestlings and ultimately their survival and future recruitment (Møller and Saino 2004; Cichon anc $\rightarrow$ Dubiec 2005; Moreno et al. 2005; López-Rull et al. 2011).

In conclusion, our study showed that in a wild population, marsh harrier nestlings' carotenoid-based traits (as coloration of bare-parts) may have not reached physiological thresholds under natural conditions; when carotenoid availability was in creased, nestling coloration increased and cell-mediated immune responsiveness to a PHA challenge also marginally increased. In nestlings of a species fed on an overal $\rightarrow$ carotenoid-poor diet, a trade-off may exist between food's energy content and that of specific nutrients (i.e., carotenoids). Differences in growth strategy may also influence differential carotenoid investment between males and females. This could explain differences between sexes in the interrelationships between condition index, carotenoid level, and immune response to PHA, possibly via access to different food as a result of intrabrood competition level and sexual size dimorphism. Finally, we suggest that the carotenoid-based coloration expressed in marsh harrier nestlings might be indicative of an offspring's quality (responsiveness to an immune challenge) rather than its current nutritional condition or need. Further work is needed, with larger sample sizes, to investigate whether carotenoid-based color expression at the nestling stage really informs about the survival prospects and ultimately the fitness of nestlings and whether it plays a significant role in parent-offspring and/or intrabrood communication.

\section{Acknowledgments}

This study was conducted under a license granted by the Centre de Recherches sur la Biologie des Populations d'Oiseaux (Muséum National d'Histoire Naturelle). This work was financially supported by the Research Group 2155 (Centre National de la Recherche Scientifique [CNRS], Groupe de Recherche en Ecologie Comportementale) "Behavioral Ecology," through a collaboration between the Centre d'Études Biologiques de Chizé (CNRS UPR1934, Chizé, France) and the University of Bourgogne (CNRS UMR5561, Dijon). A.S. was supported by a grant from the Fondation Fyssen (Paris). L.P.-R. was supported by a Juan de la Cierva postdoctoral contract from the Spanish Ministerio de Ciencia e Innovación (JCI-2008-2059). We are grateful to S. Augiron, B. Gangloff, T. Pagnon, and M. Fauchet for their dedicated help during the fieldwork. Particular thanks are due to E. Arnoux for HPLC carotenoids determination analysis (University of Bourgogne, CNRS UMR5561, Dijon) and to S. Dano and C. Trouvé for help in molecular sexing. We thank two anonymous reviewers for their helpful comments on the first draft of the manuscript.

\section{Literature Cited}

Aguilera E. and J.A. Amat. 2007. Carotenoids, immune response and the expression of sexual ornaments in male greenfinches (Carduelis chloris). Naturwissenschaften 94:895-902.

Alonso-Alvarez C., S. Bertrand, G. Devevey, M. Gaillard, J. Prosa, B. Faivre, and G. Sorci. 2004. An experimental test of the dose-dependent effect of carotenoids and immune activation on sexual signals and antioxidant activity. Am Nat 164:651-659.

$\rightarrow$ Alonso-Alvarez C. and J.L. Tella. 2001. Effects of experimental food restriction and body-mass changes on the avian T-cellmediated immune response. Can J Zool 79:101-105.

Bavoux C., G. Burneleau, and V. Bretagnolle. 2006. Gender determination in the western marsh harrier (Circus aeruginosus) using morphometrics and discriminant analysis. J Raptor Res 40:57-64. 
$\rightarrow$ Bendich A. 1991. $\beta$-carotene and the immune response. Proc Nutr Soc 50:263-274.

Benito M., J. González-Solís, and P.H. Becker. 2011. Carotenoic $\rightarrow$ supplementation and sex-specific trade-offs between coloration and condition in common tern chicks. J Comp Physiol 181:539-549.

$\rightarrow$ Biard C., P.F. Surai, and A.P. Møller. 2006. Carotenoid avail $\rightarrow$ ability in diet and phenotype of blue and great tit nestlings. J Exp Biol 209:1004-1015.

$\rightarrow$ Blanco G., O. Frías, J. Martínez, J.A. Lemus, R. Merino, and B. Jiménez. 2006. Sex and rank in competitive brood hier $\rightarrow$ archies influence stress levels in nestlings of a sexually dimorphic bird. Biol J Linn Soc 88:383-390.

$\rightarrow$ Blas J., L. Pérez-Rodríguez, G.R. Bortolotti, J. Viñuela, and T.A $\rightarrow$ Marchant. 2006. Testosterone increases bioavailability of carotenoids: new insights into the honesty of sexual signalling. Proc Natl Acad Sci USA 103:18633-18637.

$\rightarrow$ Blount J.D. and S.M. Matheson. 2006. Effects of carotenoid supply on escape flight responses in zebra finches, Taeniop $\rightarrow$ ygia guttata. Anim Behav 72:595-601.

$\rightarrow$ Blount J.D., N.B. Metcalfe, T.R. Birkhead, and P.F. Surai. 2003. Carotenoid modulation of immune function and sexual attractiveness in zebra finches. Science 300:125-127.

$\rightarrow$ Bortolotti G.R., J.M. Tella, M.G. Forero, R.D. Dawson, and J.J. Negro. 2000. Genetics, local environment and health as factors influencing plasma carotenoids in wild American kestrel (Falco sparverius). Proc R Soc B 267:1433-1438.

Brommer J.E. 2004. Immunocompetence and its costs during development: an experimental study in blue tit nestlings. Biol Lett 271(suppl. 3):S110-S113.

$\rightarrow$ Casagrande S., D. Costantini, A. Fanfani, J. Tagliavini, and G. Dell'Omo. 2007. Patterns of serum carotenoid accumulation and skin color variation in kestrel nestlings in relation to breeding conditions and different terms of carotenoid sup plementation. J Comp Physiol B 177:237-245.

$\rightarrow$ Casagrande S., D. Costantini, J. Tagliavini, and G. Dell'Omo. 2009. Phenotypic, genetic, and environmental causes of variation in yellow skin pigmentation and serum carotenoids in Eurasian kestrel nestlings. Ecol Res 24:273-279.

$\rightarrow$ Casagrande S., D. Csermely, E. Pini, V. Bertacche, and J. Tagliavini. 2006. Skin carotenoid concentration correlates with male hunting skill and territory quality in the kestrel Falc tinnunculus. J Avian Biol 37:190-196.

$\rightarrow$ Cichon M. and A. Dubiec. 2005. Cell-mediated immunity predicts the probability of local recruitment in nestling blue tits. J Evol Biol 18:962-966.

Clarke R. 1995. The marsh harrier. Hamlyn, London.

$\rightarrow$ Costantini D., C. Coluzza, A. Fanfani, and G. Dell'Omo. 2007. Effects of carotenoid supplementation on colour expression, oxidative stress and body mass in rehabilitated captive adult kestrels (Falco tinnunculus). J Comp Physiol B 177:723-731 $\rightarrow$

Cucco M., B. Guasco, G. Malacarne, and R. Ottonelli. $2006 a$. Effects of $\beta$-carotene on adult immune condition and antibacterial activity in the eggs of the grey partridge, Perdix perdix. Comp Biochem Physiol A 147:1038-1046.

$\rightarrow \longrightarrow$. 2006b. Effects of $\beta$-carotene supplementation on chick growth, immune status and behaviour in the grey partridge, Perdix perdix. Behav Processes 73:325-332.

Demas G.E., D.A. Zysling, B.R. Beechler, M.P. Muehlenbein, and S. French. 2011. Beyond phytohaemagglutinin: assessing vertebrate immune function across ecological contexts. J Anim Ecol 80:710-730.

Dubiec A., M. Cichon, and K. Deptuch. 2006. Sex-specific development of cell-mediated immunity under experimentally altered rearing conditions in blue tit nestlings. Proc $\mathrm{R}$ Soc B 273:1759-1764.

Dugas M.B. and K.J. McGraw. 2011. Proximate correlates of carotenoid-based mouth coloration in nestling house sparrows. Condor 113:691-700.

Endler J.A. 1980. Natural selection on color patterns in Poecilia reticulata. Evolution 34:76-91.

Espmark Y., T. Amundsen, and G. Rosenqvist, eds. 2000. Animal signals: signalling and signal design in animal communication. Tapir Academic Press, Trondheim, Norway.

Ewen J.G., R. Thorogood, F. Karadas, and P. Cassey. 2008. Condition dependence of nestling mouth colour and the effect of supplementing carotenoids on parental behaviour in the hihi (Notiomystis cincta). Oecologia 157:361-368.

$\rightarrow$ Faivre B., A. Grégoire, M. Préault, F. Cézilly, and G. Sorci. 2003. Immune activation rapidly mirrored in a secondary sexual trait. Science 300:103.

$\rightarrow$ Fenoglio S., M. Cucco, and G. Malacarne. 2002. The effect of a carotenoid-rich diet on immunocompetence and behavioural performances in Moorhen chicks. Ethol Ecol Evol 14: 149-156.

Fitze P.S., B. Tschirren, J. Gasparini, and H. Richner. 2007. Carotenoid-based plumage colors and immune function: is there a trade-off for rare carotenoids? Am Nat 169(suppl.): S137-S144.

Fridolfsson A.K. and H. Ellegren. 1999. A simple and universal method for molecular sexing of non-ratite birds. J Avian Biol 30:116-121.

Goodwin T.W. 1984. The biochemistry of the carotenoids. Vol. 2. Animals. Chapman \& Hall, London.

$\rightarrow$ Goto N., H. Kodama, K. Okada, and Y. Fujimoto. 1978. Suppression of phytohemagglutinin skin response in thymectomised chickens. Poult Sci 57:246-250.

$\rightarrow$ Hill G.E. 1992. Proximate basis of variation in carotenoid pigmentation in male house finches. Auk 109:1-12.

- 2002. A red bird in a brown bag: the function and evolution of ornamental plumage coloration in the house finch. Oxford University Press, Oxford.

$\rightarrow$ Hill G.E., R. Montgomerie, C. Inouye, and J. Dale. 1994. Influence of dietary carotenoids on plasma and plumage color in the house finch: intra- and intersexual variation. Funct Ecol 8:343-350.

Loiseau C., S. Fellous, C. Haussy, O. Chastel, and G. Sorci. 2008. Condition-dependent effects of corticosterone on a carotenoid-based begging signal in house sparrows. Horm Behav 53:266-273.

$\rightarrow$ López-Rull I., P. Celis, C. Salaberria, M. Puerta, and D. Gil. 2011. Post-fledging recruitment in relation to nestling plasma 
testosterone and immunocompetence in the spotless starling $\rightarrow$ Mougeot F., L. Perez-Rodriguez, N. Sumozas, and J. Terraube. Funct Ecol 25:500-508.

$\rightarrow$ Lozano G.A. 1994. Carotenoids, parasites, and sexual selection. Oikos 70:309-311. 2009. Parasites, condition, immune responsiveness and carotenoid-based ornamentation in male red-legged partridge Alectoris rufa. J Avian Biol 40:67-74.

$\rightarrow$ Martin L.B., P. Han, J. Lewittes, J.R. Kuhlman, K.C. Klasing $\rightarrow$ Mougeot F., S.M. Redpath, F. Leckie, and P.J. Hudson. 2003. and M. Wikelski. 2006. Phytohaemagglutinin-induced skin swelling in birds: histological support for a classic immunoecological technique. Funct Ecol 20:290-299.

The effect of aggressiveness on the population dynamics of a territorial bird. Nature 421:737-739.

$\rightarrow$ Navara K.J. and G.E. Hill. 2003. Dietary carotenoid pigments and immune function in a songbird with extensive carotenoid-based plumage colouration. Behav Ecol 14:909-916. Bortolotti. 2007. Nematode parasites reduce carotenoidbased signalling in male red grouse. Biol Lett 3:161-164.

$\rightarrow$ McGraw K.J. and D.R. Ardia. 2003. Carotenoids, immunocompetence, and the information content of sexual colors: an experimental test. Am Nat 162:704-712.

$\rightarrow$ Olson V.A. and I.P.F. Owens. 1998. Costly sexual signals: are carotenoids rare, risky or required? Trends Ecol Evol 13:510514.

$\rightarrow \longrightarrow$. 2007. Do carotenoids buffer testosterone-induced immunosuppression? an experimental test in a colourful songbird. Biol Lett 3:375-378.

$\rightarrow$ Pérez-Rodríguez L. 2008. Carotenoid-based ornamentation as a dynamic but consistent individual trait. Behav Ecol Sociobiol 62:995-1005.

$\rightarrow$ McGraw K.J., A.J. Gregory, R.S. Parker, and E. Adkins-Regan. 2003. Diet, plasma carotenoids, and sexual coloration in thı $\rightarrow$ Pérez-Rodríguez L., F. Mougeot, C. Alonso-Alvarez, J. Blas, J. zebra finch (Taeniopygia guttata). Auk 120:400-410.

McGraw K.J. and G.E. Hill. 2006. Mechanics of carotenoidbased colouration. Pp. 177-242 in G.E. Hill and K.J. McGraw, eds. Bird colouration. Vol. 1. Mechanisms and measurements. Harvard University Press, London.

$\rightarrow-$. 2009. Carotenoids in evolutionary ecology: re-evaluating the antioxidant role. BioEssays 31:1116-1126. Viñuela, and G.R. Bortolotti. 2008. Cell-mediated immune activation rapidly decreases plasma carotenoids but does not affect oxidative stress in red-legged partridges (Alectoris rufa). J Exp Biol 211:2155-2161.

$\rightarrow$ Saino N., R. Ambrosini, R. Martinelli, P. Ninni, and A.P. Møller. 2003a. Gape coloration reliably reflects immunocompetence of barn swallow (Hirundo rustica) nestlings. Behav Ecol 14: $16-22$. J Comp Physiol B 181:269-275.

$\rightarrow$ Millon A., B.E. Arroyo, and V. Bretagnolle. 2008. Variable bu $\rightarrow$ predictable prey availability affects predator breeding success: natural versus experimental evidence. J Zool (Lond) 275: 349-358.

Saino N., V. Bertacche, A. Bonisoli Alquati, M. Romano, and D. Rubolini. 2008. Phenotypic correlates of yolk and plasma carotenoid concentration in yellow-legged gull chicks. Physiol Biochem Zool 81:211-225.

Mock D.W. and G.A. Parker. 1997. The evolution of siblin $\rightarrow$ Saino N., S. Calza, and A.P. Møller. 1997. Immunocompetence rivalry. Oxford University Press, Oxford.

Møller A.P., C. Biard, J.D. Blount, D.C. Houston, P. Ninni, N. Saino, and P.F. Surai. 2000. Carotenoid-dependent signals $\rightarrow$ Saino N., R. Ferrari, M. Romano, R. Martinelli, and A.P. Møller. indicators of foraging efficiency, immunocompetence or detoxification ability? Avian Poult Biol Rev 11:137-159.

$\rightarrow$ Møller A.P. and N. Saino. 2004. Immune response and survival. Oikos 104:299-304.

$\rightarrow$ Moreno J., S. Merino, J.J. Sanz, E. Arriero, J. Morales, and G. Tomas. 2005. Nestling cell-mediated immune response, body mass and hatching date as predictors of local recruitment in the pied flycatcher Ficedula hypoleuca. J Avian Biol 36:251260. of nestling barn swallows in relation to brood size and parental effort. J Anim Ecol 66:827-836. 2003b. Experimental manipulation of egg carotenoids affects immunity of barn swallow nestlings. Proc R Soc B 270:24852489.

$\rightarrow$ Saino N., P. Ninni, S. Calza, R. Martinelli, F. De Bernardi, and A.P. Møller. 2000. Better red than dead: carotenoid-based mouth colouration reveals infection in barn swallow nestlings. Proc R Soc B 267:57-61.

SAS. 2001. SAS/STAT user's guide. Version 8.01. SAS Institute, Cary, NC.

$\rightarrow$ Mougeot F. 2008. Ornamental comb colour predicts T-cell $\rightarrow$ Smits J.E., G.R. Bortolotti, and J.L. Tella. 1999. Simplifying the mediated immunity in male red grouse Lagopus lagopus scoticus. Naturwissenschaften 95:125-132.

$\rightarrow$ Mougeot F. and B.E. Arroyo. 2006. Ultraviolet reflectance b; $\rightarrow$ S the cere of raptors. Biol Lett 2:173-176.

$\rightarrow$ Mougeot F., J. Martínez-Padilla, G.R. Bortolotti, L.M.I. Webster, and S.B. Piertney. 2010. Physiological stress links parasites to carotenoid-based colour signals. J Evol Biol 23:643-650.

$\rightarrow$ Mougeot F., J. Martínez-Padilla, L. Pérez-Rodríguez, and G.R. Bortolotti. 2007. Carotenoid-based colouration and ultraviolet reflectance of the sexual ornaments of grouse. Behav Ecol Sociobiol 61:741-751. phytohaemagglutinin skin-testing technique in studies of avian immunocompetence. Funct Ecol 13:567-572.

Soler J.J., L. de Neve, T. Pérez-Contreras, M. Soler, and G. Sorci. 2003. Trade-off between immunocompetence and growth in magpies: an experimental study. Proc R Soc B 270:241-248. Sternalski A., J.F. Blanc, S. Augiron, V. Rocheteau, and V. Bretagnolle. 2012a. Comparative breeding performance of marsh harrier along a gradient of land-use intensification, and implications for population management. Ibis (forthcoming).

$\rightarrow$ Sternalski A., F. Mougeot, and V. Bretagnolle. 2012b. Carot- 
enoid limitation and allocation priorities in asynchronou: $\rightarrow$ Tella J.L., J.A. Lemus, M. Carrete, and G. Blanco. 2008. The raptor nestlings. Biol J Linn Soc 105:13-24.

$\rightarrow$ Sternalski A., F. Mougeot, C. Eraud, B. Gangloff, A. Villers, and PHA test reflects acquired T-cell-mediated immunocompetence in birds. PLoS ONE 3:e3295.

V. Bretagnolle. 2010. Carotenoids in nestling Montagu's harri $\rightarrow$ Thorogood R., R.M. Kilner, F. Karadas, and J.G. Ewen. 2008. ers: variations according to age, sex, body-condition and evi- Spectral mouth colour of nestlings changes with carotenoid dence for diet-related limitations. J Comp Phys B 180:33-43.

Stevens L. 1996. Avian biochemistry and molecular biology Cambridge University Press, Cambridge.

$\rightarrow$ Stradi R., G. Celentano, and D. Nava. 1995. Separation and identification of carotenoids in bird's plumage by high-performance liquid chromatography-diode-array detection. J Chromatogr B 670:337-348. availability. Funct Ecol 22:1044-1051.

Surai P.F. 2002. Natural antioxidants in avian nutrition and reproduction. Nottingham University Press, Nottingham. Tschirren B., P.S. Fitze, and H. Richner. 2003. Proximate mechanisms of variation in the carotenoid-based plumage colouration of nestling great tits (Parus major L.). J Evol Biol 16:91-100.

$\rightarrow$ Vinkler M., H. Bainova, and T. Albrecht. 2010. Functional analysis of the skin-swelling response to phytohaemagglutinin. Funct Ecol 24:1081-1086. 